Perfil ecuatoriano de las empresas metalmecánicas

\title{
Perfil Ecuatoriano de las empresas metalmecánicas
}

\section{Ecuadorian profile of the metalworking companies}

\section{Perfil ecuatoriano das empresas metalmecánicas}

María E. Jiménez-Cercado ${ }^{\mathrm{I}}$

jimenezcme@ug.edu.ec

Marco A. Navarrete-Pilacuan II

marco.navarretep@ug.edu.ec

Recibido: 31 de agosto de 2017 * Corregido: 14 de noviembre de $2017 *$ Aceptado: 17 de diciembre de 2017

I. Magister en Administración de Empresas Mención en Recursos Humanos y Marketing, Ingeniera Comercial, Docente de la Universidad de Guayaquil, Guayaquil, Guayas, Ecuador.

II. Magister en Educación Superior, Ingeniero Comercial, Docente de la Universidad de Guayaquil, Guayaquil, Guayas, Ecuador. 


\title{
Resumen
}

El presente estudio refleja un análisis del desarrollo del mercado metalmecánico en el Ecuador, desde sus inicios hasta la actualidad, abarcando las oportunidades tanto nacionales como internacionales que tiene para expandirse. Porcentajes de crecimiento y montos de inversión que ha realizado esta división son señalados, adicionalmente de las cifras de empleos directos e indirectos que brinda. Se revisan los sectores y sub-clasificaciones de los mismos en los cuales se basa la producción. Finalmente, se concluye con importantes reflexiones sobre el futuro de la industria que a pesar de ralentización, se augura crecimientos importantes para el avance productivo del país.

Palabras clave: metalmecánica; siderurgia; acero; hierro.

\begin{abstract}
The present study reflects an analysis of the development of the metalworking market in Ecuador, from its beginnings to the present, covering the national and international opportunities it has to expand. Percentages of growth and investment amounts made by this division are indicated, in addition to the figures of direct and indirect employment that it provides. It reviews the sectors and sub-classifications of the same on which the production is based. Finally, we conclude with important reflections on the future of the industry that, despite slowing down, augurs important growths for the country's productive progress.
\end{abstract}

Keywords: metalworking; steel industry; steel; iron.

\section{Resumo}

O presente estudo reflete um análise do desenvolvimento do mercado metalmecânico no Equador, desde os inícios até a atualidade, abarcando as oportunidades tanto nacionais como internacionais que tem para expandirse. Porcentajes de crescimento e montes de investimento que realizaram esta divisão e seus signatários, além de mensagens de empregos direcionados e indiretos que brinda. Se revisar os sectores e sub-clasificaciones de os mesmos em todos os meios. Finalmente, conclua com importantes reflexões sobre o futuro da indústria que é um processo de ralentização, e crie equipamentos para o desenvolvimento do país.

Palavras chave: metalmecánica; siderurgia; aço; hierro. 


\section{Introducción}

Los últimos siglos pueden considerarse como una verdadera transformación. Con la Revolución Industrial, surgen muchos cambios en la humanidad, comparándose así con la tecnología que hoy se vive. Uno de estos cambios es el aumento de producción de acero y de hierro, con lo cual tanto mundialmente como localmente, se ha generado un boom en el sector de la construcción y demás sectores que tienen como materia prima los elementos ya mencionados, ocasionando así un gran desarrollo de la economía global desde entonces hasta los tiempos actuales. (Tendenzias, 2017)

Los cambios culturales que se plasmaron a inicios del siglo XIX, se destacaron por el crecimiento de las ciudades y por consiguiente el incremento del éxodo de personas provenientes de zonas rurales. Este y otros factores conllevan a que la base de la nueva civilización sea el hierro, el mismo se considera como el metal dominante, desplazando a la madera en los trabajos de los arquitectos. El hierro fundido muy tempranamente fue utilizado para la creación de edificios y puentes de forma masiva.

El consumo del hierro en el mundo ha crecido vertiginosamente al pasar de los años, siendo su ventaja el ser 95 de cada 100 de los minerales extraídos de la tierra, actualmente y gracias a las nuevas técnicas siderúrgicas también puede mezclarse o fundirse con gran parte de otros minerales entre ellos níquel y el cromo. Fue a partir de la revolución industrial que el perfeccionamiento de las técnicas de construcción ha permitido el desarrollo de aleaciones más eficientes inclusive en el ámbito espacial. (Historia de la Siderurgia minerales de hierro obtención del acero, 2014)

Antiguamente al proceso se lo denominó ‘carburación' del hierro, que consistía en la unión de cierta cantidad de carbono. Las primeras técnicas de fundición, cuya fecha exacta de inicio de uso se desconoce, evolucionan a nivel mundial por la creación de altos hornos, los mismos que surgen a partir de los años 1800. Es entonces, y con la finalidad de tornar esta actividad en un proceso más eficiente y limpio, se crean nuevas tecnologías como el horno de arco eléctrico, plantas de humo, crisol abierto, sistema consteel, etc.

Gracias al incremento de esta actividad, las economías de los países del globo, en especial las potencias, crecieron y continuamente estuvieron en capacidad de renovar sus técnicas de construcción. Países como Japón, Corea, China y Taiwán son líderes del sector, ya que llegan a más mercados debido a que producen más variedades de productos. 
En Latinoamérica, la producción de hierro y acero ingresa por motivo del desabastecimiento que dejó la posguerra, es decir al finalizar la segunda guerra mundial. Por consiguiente ocasionó que en América Latina se sustituya la importación del elemento y así empezara su propia producción de acero especialmente largo y no plano. El sector de la construcción amplió su demanda, generando interés en otras áreas como las de tecnología, servicio y transporte conforme avanzaba la variedad que la industria metalmecánica podía ofrecer.

Por un lado el hierro puede ser extraído de la naturaleza, mientras que por otro lado, puede ser obtenido del reciclaje, éste último ha ocasionado una verdadera revolución para la industria del hierro. El reciclaje de los metales contribuye de manera importante a no empeorar el entorno medioambiental. (Rodríguez, 2007). Lo que se recicla es la chatarra, por ejemplo, el 70 por ciento de reducción de desechos de minería ocasiona también que reduzca la contaminación del aire y del agua

Otro ejemplo claro es que el $60 \%$ de la producción nueva de hierro en Norteamérica es a base de chatarra, generando en productos reciclados tales como: motores, automóviles, latas de bebidas, instrumentos musicales, aviones, bicicletas, artículos de consumo y caseros, ingresos por más de 30.000 millones de dólares. Es claro destacar que entre los beneficios del reciclaje están la reducción del impacto ambiental por la extracción de sus minerales y, los procesos de reciclaje del acero encontrado en la chatarra son infinitamente menos nocivos que producir acero nuevo.

El liderazgo latinoamericano lo tienen México y Brasil, considerando a la compañía GERDAU como la empresa recicladora brasileña de mayor expansión. Solo en el 2012 toda su producción la basó en reciclaje, la cual fue de 900.000 toneladas de chatarra, el $40 \%$ de agua y $80 \%$ de energía se ahorra en un proceso de reciclase frente a un proceso regular de extracción. Los residuos metálicos entre ellos los sobrantes industriales como recortes y virutas, restos de demolición de estructuras, desguaces de autos y electrodomésticos, son aquellos de los principales elementos considerados chatarra y que han podido cubrir el 100\% de la producción de Gerdau. (Gerdau, 2016)

El hierro y el acero son materiales considerados eficientes, en el sentido que pueden ser reciclados varias veces sin que pierdan sus propiedades, en comparación con otros elementos. En tal sentido, también surge el compromiso ambiental de las empresas y de los países por mantener un mejor ecosistema, tal es el caso de España que a partir de los años 70, ha podido reducir emisiones $\mathrm{CO} 2$ en 


\section{Perfil ecuatoriano de las empresas metalmecánicas}

un $75 \%$, y en ese mismo porcentaje y más es la producción de acero reciclado frente a un promedio de $50 \%$ europeo y $40 \%$ mundial.

Además de ser líder en proveer aceros largos en el continente americano, Gerdau es uno de los principales proveedores de este elemento en el mundo, posee más de 45 mil trabajadores operando en más allá de 14 países en América, Europa y Asia, con una capacidad instalada mayor a 25 millones de toneladas al año. Es por esto que Gerdau es la recicladora más grande de América Latina y también transforma millones de toneladas de chatarra en acero alrededor del mundo. Cuenta con más de 140 mil accionistas, adicionalmente se encuentra enlistada en la bolsa de valores de prestigio como son las de Sáo Paulo, Nueva York y Madrid.

En Ecuador, según el Periódico El Universo (2014), la Empresa Nacional Minera ENAMI anunció que existen indicios de que existe un potencial yacimiento de arenas ferrotitaníferas, base para la producción de hierro, en el proyecto Tola Norte situado en Esmeraldas y que fue enviado al Instituto Nacional de Investigación Geológico Minera Metalúrgico para su respectivo análisis. El proyecto contempla la exploración avanzada que incluye el proceso de obtención de la licencia ambiental para desarrollar el yacimiento. (El Universo, 2014)

No obstante, Ecuador no cuenta con minas de hierro donde se extraiga, se haga la reducción y se obtenga el producto base para la producción de todos los derivados del acero. Por tanto importa su materia prima y utiliza el proceso de reciclaje a través de la chatarra proveniente de los materiales ricos en contenido de hierro como barcos, línea automotriz, línea blanca, entre otros desechos industriales y domésticos. (Acosta \& Jiménez, 2016)

En 1963, un grupo de empresarios ecuatorianos creó ADELCA, la primera acería del Ecuador, pionera en el reciclaje y fabricación de acero. Además de operar bajo estrictas normas técnicas y de seguridad industrial, Adelca tiene un eficiente programa de conservación ambiental. Adicionalmente, abastece con su producción a sectores como la construcción, metalmecánico, agroindustria y seguridad perimetral. (Adelca, 2017)

Un año después, en 1964, dado al gran desarrollo industrial y sobre todo del sector de la construcción se crea ANDEC Acerías Nacionales del Ecuador, la cual inició con 150 trabajadores quienes se capacitaron en el uso de un tren laminador que funcionó hasta los ochenta, generando en 1970 una 
Perfil ecuatoriano de las empresas metalmecánicas

producción de 11.250 toneladas. Obtuvo la certificación ISO 9000 en 1997 y gracias a las diversificaciones y nuevos procesos de Gestión de Calidad logró obtener la certificación ISO 9002 en el 2001, actualmente, cuenta con las normas ISO 14001 y OHSAS 18001. (Andec, 2014)

Es así y gracias a la buena acogida se destacan Adelca y Andec como las empresas líderes en el mercado del hierro en el Ecuador. Ya para el año 1973, un grupo de inversionistas crean la empresa NOVACERO, la cual empezó con el negocio de productos viales para luego expandirse a otra gama de productos. Entre las tres empresas se comparten la mayor participación en el Ecuador, del mercado metalmecánico.

En el sector metalmecánico se encuentran diversas empresas las cuales importan, distribuyen y también comercializan los productos elaborados y semi-elaborados provenientes del exterior, algunas de ellas son: Rooftec, Dipac, Ideal Alambrec, Kubiec y Tugalt. Es necesario mencionar que el sector genera no solo 23600 empleos directos sino también más de 50000 empleos indirectos, siendo por esta razón considerado dentro del país como uno de los sectores primarios para el desarrollo y de carácter transversal por la influencia que ejerce en otras áreas industriales y productivas, dados los productos que se obtienen del mismo como lo son para la construcción, automotriz, carrocerías y maquinarias en general. (Proecuador, 2013)

Es por esto que el sector metalmecánico actualmente influye en más del 10\% de la industria ecuatoriana, generando una cobertura más amplia al pasar del tiempo, su análisis es trascendente dado que su participación en el mercado actual augura pronósticos excelentes para el futuro, destacando una visión optimista acompañada de temas ecológicamente amigables.

\section{Metodología}

Este trabajo es exploratorio, es decir que provee una referencia general de la temática, a menudo desconocida, presente en la investigación a realizar, tal es el caso del mercado metalmecánico en el Ecuador y entre sus propósitos se encuentra la extracción de datos y términos que dan apoyo a la investigación descriptiva. (Hernández Sampieri \& Fernández Collado, 2003), utiliza datos de la historia así como datos bibliográficos. (Morales, 2016)

Es de carácter descriptivo, método científico en el cual se describe y se observa el comportamiento del objeto de estudio sin influir sobre este de ningún modo. (Shuttleworth, 2008), en otras palabras 
permite la observación sin afectar su procedimiento normal. Sus resultados no pueden ser considerados una respuesta definitiva sino más bien reflejan una base para futuras investigaciones.

Adicionalmente, es cualitativa, cuya finalidad es recoger la información basado en la observación de comportamientos naturales, discursos, respuestas abiertas para su posterior interpretación. (Ibañez, 1992). Básicamente se puede definir que la investigación cualitativa se basa en una observación constante lo que permite el análisis de cada situación relacionado con los procedimientos de las personas o el objeto de estudio, tal como: el entorno, experiencias, conocimientos y contextos.

Asimismo, a través de la metodología empírica conjuntamente con los métodos teóricos de análisis y síntesis, inducción y deducción, se recaba información tanto histórica como también lógica que tenga como objetivo principal recoger las consecuencias y su relación no solamente para descubrir sino construir el conocimiento.

\section{Desarrollo}

Para el desarrollo del trabajo es importante conocer los términos básicos y conceptos respecto al tema a tratar, entre los cuales están:

- Metalmecánica: la industria es un conjunto de operaciones de obtención y transformación de materias primas, también se define al metal como cada uno de los elementos químicos que se caracterizan por ser sólidos y buenos conductores del calor y la electricidad por ejemplo el hierro. Es por tanto, que metalmecánica es un proceso de diseño y fabricación de estructuras metálicas, aunque el concepto es sencillo los procesos y calidad son muy complejos, ya que implica un proceso un conjunto de diversas acciones donde se utilizan productos de la siderurgia empleando algún tipo de transformación, ensamblaje o reparación. (Reverso Diccionario, 2017)

- Siderurgia: es un sector de la industria del metal dedicado a la extracción de hierro, su sinónimo es "Acería", empresa de este sector de la industria del metal. En la figura 1 se hace alusión que actualmente la siderurgia no afecta más al medio ambiente. 


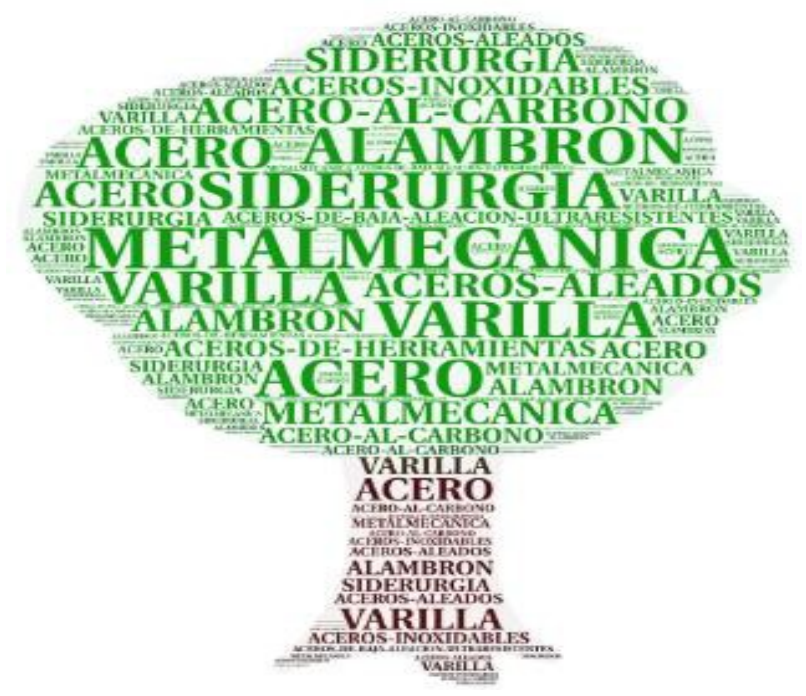

Figura 1: Nube de Palabras. Fuente: Elaboración en Software Word Art

- Varilla: barra fina y larga, en especial la que es un componente de la estructura o forma la armazón de un objeto, generalmente es de metal o de madera.

- Alambrón: el alambre es un hilo de metal, mientras que el alambrón es un producto derivado de metal sometido a un proceso de laminación en caliente, es de sección redonda y macizo, se lo encuentra en diferentes presentaciones de acuerdo a su diámetro que puede ir desde $4.5 \mathrm{~mm}$ hasta $30 \mathrm{~mm}$.

- Acero: es básicamente una aleación o combinación de hierro y carbono. El hierro puro es uno de los elementos del acero. Entre los principales tipos de acero se destacan cinco: aceros al carbono, aceros aleados, aceros de baja aleación ultrarresistentes, aceros inoxidables y aceros de herramientas. (Encarta, 2017)

- Aceros al carbono: la mayoría estimando 90\% de la producción de acero, corresponde a acero de carbono, su composición se basa en carbono, en tanto que menos del 1.65\% es de manganeso, el $0.60 \%$ de silicio y el $0.60 \%$ de cobre. Entre los principales productos que se fabrican con este componente son: gran parte de las estructuras de construcción de acero, carrocerías de automóvil, cascos de buque, máquinas, somieres y pasadores u horquillas para el cabello. 
- Aceros aleados: este tipo de aceros contienen una proporción determinada de vanadio, molibdeno y otros elementos, adicionalmente posee en mayor cantidad otros elementos como manganeso, silicio y cobre en comparación con los aceros de carbono regulares. Puede sub-clasificarse en los siguientes:

- Aceros estructurales: sirven como base para partes de máquinas como por ejemplo los engranajes, ejes y palancas, adicionalmente se utilizan en la construcción de estructuras de edificios, chasis de automóviles, barcos, puentes y semejantes. La variación del contenido de la aleación se encuentra entre un $0.25 \%$ a un $6 \%$.

- Aceros para herramientas: este se considera un acero de alta calidad ya que se usa en la elaboración de herramientas para cortar y modelar metales y no metales, entre ellas están: los taladros, fresas, escariadores, machos de roscar y terrajas.

- Aceros especiales: se los conoce también como aceros inoxidables, poseen cromo en una cantidad mayor al $12 \%$, por su alta resistencia a altas temperaturas y dureza contra la corrosión, puede ser utilizado en rodamientos, ejes, engranajes y turbinas de vapor.

- Aceros de baja aleación ultrarresistentes: de los cuatro grupos, este es el más reciente y también menos costoso, ya que contiene menor componentes de los más económicos usados en su aleación. $\mathrm{Su}$ resistencia es mucho mayor que el acero de carbono debido al tratamiento especial que se le da, esto permite a su vez en el caso de vagones, transportar más carga dado que sus paredes delgadas proporcionan más espacio y al ser más liviana también admite carga más pesada; de igual manera, las vigas delgadas son utilizadas en los edificios modernos por su flexibilidad y resistencia también logrando más espacio interior.

- Aceros inoxidables: estos contienen níquel, cromo y demás elementos para alearse, con la finalidad de mantenerse brillantes y resistentes a la herrumbre, óxido, humedad y corrosión. $\mathrm{Su}$ resistencia y su dureza le permiten durar por largos períodos de tiempo y a temperaturas extremas, de hecho, tiene fines decorativos especialmente en la arquitectura. Adicionalmente se utiliza para la elaboración de tanques de refinerías de petróleo o plantas químicas, tuberías, fuselajes de los aviones y hasta capsulas espaciales; en medicina, se usa en la producción de equipos quirúrgicos, sustituto de huesos rotos debido a que resiste a la acción de los fluidos corporales; en la cocina, se 
encuentran en los utensilios ya que por ser de acero inoxidable preserva los alimentos y son de fácil limpieza.

- Aceros de herramientas: SAE es la abreviatura en inglés para Society of Automotive Engineers (Sociedad de Ingenieros Automotores), es también conocida AISI-SAE cuyo acrónimo es American Iron and Steel Institute (Instituto Americano de hierro y acero), ambas promueven el intercambio de informaciones y propuestas de unificación de los materiales y las normas entre las diferentes industrias adicionalmente a la automovilística (Motorgiga, 1998), en otras palabras, es la norma que regula la calidad del acero velando por su correcta clasificación y aleación de materiales no ferrosos. En tal sentido, la SAE clasifica el acero para herramientas en los siguientes grupos: $\mathrm{W}$ de temple en agua, $\mathrm{S}$ resistentes al impacto, O.A.D. para trabajo en frío, $\mathrm{H}$ para trabajo en caliente, T.M. rápidos y L para propósitos especiales. Es decir, que sirven para fabricar muchos tipos de herramientas y máquinas. (Aceros de Herramientas, s/f)

Los productos ecuatorianos se clasifican de acuerdo al siguiente detalle:

- Metálicas básicas

- Productos Metálicos

- Maquinarias no eléctricas

- Maquinarias eléctricas

- Material de Transporte y carrocería

- Bienes de capital

Así mismo, existen más productos provenientes de los subsectores metalmecánicos:

- Cubiertas Metálicas

- Tuberías

- Perfiles Estructurales

- Perfiles Laminados

- Invernaderos viales

- Sistemas Metálicos

- Varilla de Construcción

- Alcantarillas 
- Productos viales

- Señalización y

- Línea blanca

Conjuntamente con los productos detallados, se adiciona aquellos productos que se utilizan como insumo en el sector petrolero, ya sea para la fabricación de maquinarias así como también para equipos, de igual manera la cobertura se amplia para los sectores claves de desarrollo como la agroindustria y el sector eléctrico. (Proecuador, 2013)

Es importante destacar que los mercados internacionales son una gran amenaza, tal es el caso de China, que siendo el mayor productor del mundo, vende su producción al costo. Sin embargo, Ecuador ha superado los efectos de la globalización como lo son los bajos precios por ejemplo y mantiene su fabricación local. En la figura 2 se detalla la producción del país en toneladas.

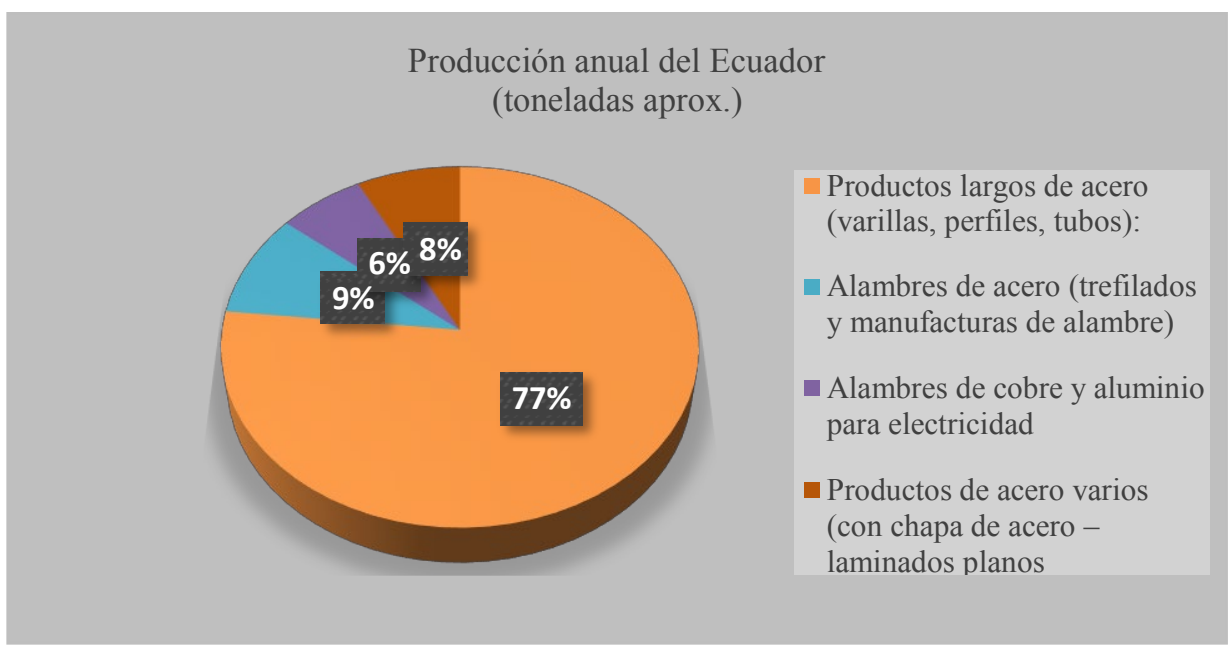

Figura 2: Producción Anual del Ecuador (toneladas aprox.) Fuente: Pro Ecuador 2016

\section{Perspectiva al futuro}

El clima de inversiones en Ecuador se considera bueno dado la estabilidad económica que el país ofrece por los últimos 8 años, siendo el PIB Producto Interno Bruto de 1.5\% en esta industria con atención al endeudamiento bajo y alto crecimiento que según el CEPAL Comisión Económica para América Latina, sería del 1.9\% a pesar de la recesión económica que atravesó el país y que en realidad fue del 1.7\%, aun así se encuentra superior al promedio regional que es de 0.5\%. (Economía, El sector Metalmecánico espera por más inversiones, 2015) 


\section{Perfil ecuatoriano de las empresas metalmecánicas}

La inversión que se augura para el sector metalmecánico es de USD 624 millones, a pesar de una contracción del 4\% dada las dificultades económicas que sufrió el sector de la construcción. Tal es el caso de la empresa Adelca que en abril de este año invita a la apertura de una nueva planta de producción ubicada en la ciudad de Milagro, a 40 kilómetros de Guayaquil, la misma que tiene una extensión de 70 hectáreas acogiendo a 1540 colaboradores a escala nacional. Este proyecto que inició en el 2012, tiene una inversión aproximada de 130 millones de dólares, siendo uno de sus objetivos sustituir la importación de alambrón de acero, de este modo contribuye a la balanza comercial con más de USD100 millones. (Acería del Ecuador C.A. Adelca - Planta Miilagro, 2017)

Considerando que la demanda en Ecuador es de 1.5 millones de toneladas y que la producción local es de 900 mil, se evidencia la confianza en el sector y aunque las empresas no han cerrado como en otros segmentos de mercado, han tenido que adaptarse a las circunstancias reduciendo personal y utilizando la más alta tecnología para entregar al mercado los productos que las técnicas modernas de construcción y fabricación demanden, de tal suerte que se busca inversión en la producción de bobinas para la elaboración de materia prima que fabrica productos planos de acero como tuberías y planchas, artes y piezas para el ensamblaje de vehículos local, algo en lo que se ha avanzado en los últimos años con la producción nacional de amortiguadores, y finalmente equipos para riego tecnificados. (Economía, Ecuador supera la recesión económica y cerrará el 2017 con crecimiento del 1,42\%, 2017)

Como se mencionó anteriormente los procesos actuales requieren tecnificarse, a esto se le denomina tendencia verde, por tanto cuidar la naturaleza es prioridad en los actuales intereses de los clientes, entre los principales productos requeridos son: nuevo estándar de hierro modular EN1563, aplicaciones innovadoras de aluminio y magnesio en el coste de fundición de hierro, estructuras metálicas, artículos de ferretería y sistemas constructivos. El estado ecuatoriano a través de su Ministerio del Ambiente, otorga la certificación Punto Verde que se suma al otorgado por el Sistema de Gestión Ambiental.

El sector metalmecánico genera empleo en el país de acuerdo al INEC Instituto Nacional de Estadísticas y Censos, un 65\%, mientras que su crecimiento en un período de 10 años es del 7\%. El Encadenamiento Productivo, lo cual es una ventaja de esta industria, supera gracias al consumo de acero que es del 65\% frente al sector manufacturero cuyo porcentaje es del 59\%. (Proecuador, 2013). 
Siendo Guayas, Pichincha, Azuay, Tungurahua y Loja, las provincias en donde se desarrollan más esta actividad.

La excelente calidad y competitividad del producto ecuatoriano, le ha permitido competir en otros países de la región, llegando a casi 500 millones de dólares sus exportaciones. Los cinco primeros países a los cuales se exporta son: Venezuela, Colombia, Perú, China y Estados Unidos.

En la tabla 1 se puede apreciar los principales países conjuntamente con su valor FOB (Free on Board), número de toneladas y cuál es su participación al año 2012:

\begin{tabular}{llll}
\hline País & Valor FOB & Toneladas & \% Participación \\
\hline Venezuela & 116.809 & 14.512 & $23.63 \%$ \\
Colombia & 92.652 & 29.957 & $18.75 \%$ \\
Perú & 61.595 & 20.071 & $12.46 \%$ \\
China & 55.178 & 13.399 & $11.16 \%$ \\
Estados Unidos & 38.156 & 6.561 & $7.72 \%$ \\
\hline
\end{tabular}

Tabla No. 1 Países a los que Ecuador Exporta Fuente: Proecuador

Las exportaciones ecuatorianas del sector metalmecánico tiene como cinco primeros productos: 1. Desperdicios y desechos de cobre, el mismo que se exporta a China teniendo una participación de 9.03\%. 2. Cocinas de combustibles gaseosos a Venezuela, cuya participación es 6.66\%. 3. Cocinas de combustibles gaseosos a Perú, su participación es 4.81\%. 4. Cocinas de combustibles gaseosos hacía Colombia siendo su participación de 3.76\%. Y, 5. Máquinas o aparatos para tratamiento de arroz exportado a Venezuela y su participación es 3.03\%

En el Gráfico 1 se puede apreciar las exportaciones por productos principales de los años 2010, 2011 y 2012, cuya fuente es el Banco Central del Ecuador, un incremento en los valores FOB de los cuatro productos detallados destacando que las exportaciones sí tienen tendencias al alza. Los principales países exportadores son: China, Alemania, Estados Unidos, Japón y Hong Kong. En cuanto a los países importadores se destacan los primeros cinco: Estados Unidos, China, Alemania, Hong Kong y Japón. (Proecuador, 2013) 
Perfil ecuatoriano de las empresas metalmecánicas

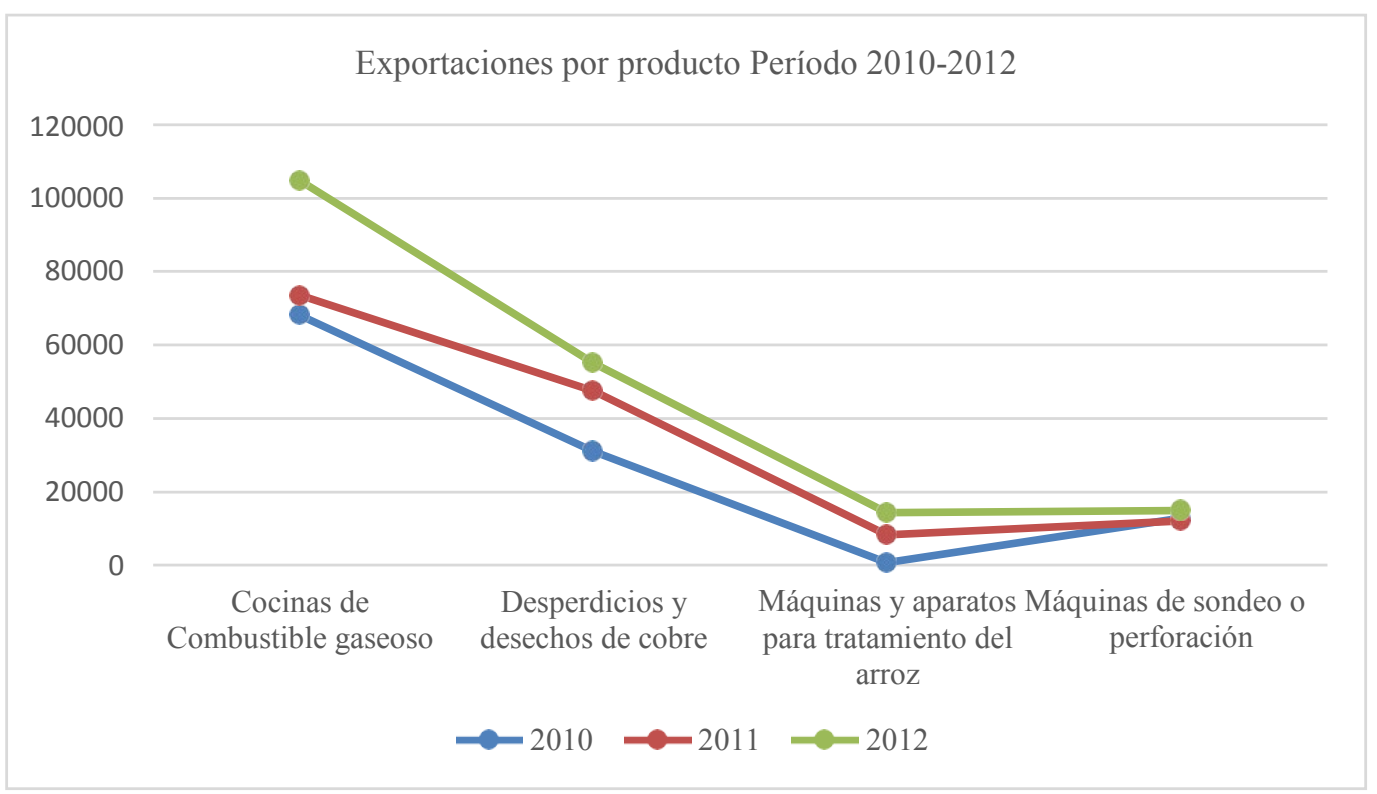

Gráfico 1: Principales productos exportados período 2010-2012 Fuente: Proecuador

A continuación se detalla las empresas procesadoras de acero según la clase de producto:

- Laminados: ADELCA, ANDEC, NOVACERO, TALME.

- Tubería: IPAC, NOVACERO, CONDUIT, TUGALT, DIPAC, FERROTORRES.

- Perfiles: IPAC, NOVACERO, DIPAC, KUBIEC, FERRMOTORES.

- Cubiertas: ROOFTEC, KUBIEC, DIPAC, TUGALT, NOVACERO.

- Trefilados: IDEAL ALAMBREC, ADELCA, TREFILEC

Cabe recalcar que una de las estrategias de marketing exitosas ha sido la alianza específicamente en el sector de la construcción, tal es el caso de DISENSA, franquicia que se encuentra en todo el territorio nacional, beneficiando además del sector metalmecánico, a otras industrias relacionadas como cemento y tuberías plásticas. El crecimiento de las empresas dedicadas a este sector del $2010 \mathrm{al}$ 2013 fue del 19,6\%, (Garzón \& Matías, 2016) es el mayor de los últimos años, generando por sí sola $4.4 \%$ del total de las ventas de las empresas ecuatorianas.

\begin{tabular}{lll}
\hline Descripción & \% Total & Empleo \\
\hline $\begin{array}{l}\text { Fabricación de productos elaborados de metal, excepto } \\
\text { maquinaria y equipo }\end{array}$ & $4,3 \%$ & 13.186 \\
Fabricación de metales comunes & $2,3 \%$ & 6.894 \\
Fabricación de maquinaria y equipo & $1,1 \%$ & 3.507 \\
\hline
\end{tabular}

Tabla 2: Rubros de la industria manufacturera con mayor crecimiento de empleo Fuente: INEC 
De un total de 10 rubros, se puede apreciar en la tabla 2 que la industria del metal suma una participación de 7.7\%, llegando a generar empleos por más de 23 mil. Sin contar que para otros productos también está involucrado el metal y el acero, tal es el caso de maquinarias y equipos para la fabricación de productos minerales no metálicas, bebidas y prendas de vestir que sumadas dan una participación de $15,3 \%$.

Los principales sectores en donde se goza de más prestigio y presencia comercial son: 1. El sector de la construcción y obras de infraestructura. 2. Sector Primario, Secundario y Terciario que involucra inversiones por adquisición de equipos y maquinaria. 3. Bienes de consumo final así como consumo durable y, 4. La industria automotriz y material de transporte en general (Fedimetal, 2014)

Con la finalidad de seguir creciendo, es necesario que el sector metalmecánico se enfoque en trabajar de manera colaborativa con el sector público, de modo que pueda sostener el 3-6\% de incremento en la industria, seguir captando inversiones que le permitan desarrollar sus actividades oportunamente, que den valor agregado a su producción y así abastecer el mercado nacional plenamente y aumentar sus exportaciones.

Entre las iniciativas estratégicas se encuentran las siguientes:

- Requerimientos que el sector tiene para el Gobierno

- Normas técnicas y estándares de calidad

- Fuentes de Financiamiento

- Políticas arancelarias y salvaguardias

- Gestión comercial en mercados internacionales

- Requerimiento que el sector tiene para la Academia

- Inventario de necesidades del país

- Inventario de necesidades del sector 2020

\section{Conclusiones}

El sector metalmecánico es una industria que puede seguir desarrollándose de manera exitosa, especialmente si asume los retos que el mundo globalizado le da, el tema tecnológico hoy en día hace la diferencia y Ecuador no puede quedarse atrás, es así que los tiempos modernos requieren una producción más competitiva y moderna. 


\section{Perfil ecuatoriano de las empresas metalmecánicas}

Al igual que otros sectores, los especialistas en el tema aportan con ideas y soluciones para conllevar de manera conjunta desafíos constantes tales como ampliar mercados tanto nacionales como internacionales, mantener y fomentar diálogos entre asociados privados y autoridades públicas. La Federación Nacional de la Industria Metalmecánica es un ejemplo de aquello.

El análisis del sector permitió dar un panorama general de la importancia que tiene la industria metalmecánica en cuanto al desarrollo del país dado los porcentajes de crecimiento para la economía y balanza comercial, pero sobre todo a la generación de empleos directos e indirectos que el engranaje productivo proporciona.

Las estrategias de mercadeo son una elección acertada que aporta a la producción nacional, competitividad además de valor agregado, a fin de consolidar las alianzas de modo que las exportaciones puedan mantenerse y por qué no ampliarse a otros países de la región y del mundo.

\section{Referencias Bibliográficas}

Acería del Ecuador C.A. Adelca - Planta Miilagro. (2017). Revista Ekos.

Aceros de Herramientas. (s/f). Recuperado el 30 de Mayo de 2017, de Universidad Tecnológica Nacional: https://www.frro.utn.edu.ar/repositorio/.../17-_Aceros_de_herramientas_(frio)_v2.pd

Acosta, M., \& Jiménez, M. (2016). Desarrollo del mercado metalmecánico en el Ecuador. Ier Congreso de Marketing: El marketing del siglo XXI, 244-254.

Adelca. (2017). Adelca Acería del Ecuador. Obtenido de http://www.adelca.com/

Andec. (2014). ANDEC La fuerza Interior del Ecuador. Obtenido de https://www.andec.com.ec/index.php/es/Conocenos/historia

Economía. (29 de Julio de 2015). El sector Metalmecánico espera por más inversiones. El Telégrafo.

Economía. (8 de Abril de 2017). Ecuador supera la recesión económica y cerrará el 2017 con crecimiento del 1,42\%. El Telégrafo.

El Universo. (23 de Marzo de 2014). Potencial área con hierro se reporta en Esmeraldas.

Encarta, 2. (2017) ¿Qué es el Acero? InfoAcero. Santiago, Chile. 
Perfil ecuatoriano de las empresas metalmecánicas

Fedimetal. (2014). Política, Estrategias y Plan de Acción 2035 para la Industria Metalmecánica Ecuatoriana. Construmetal, 13-14.

Garzón, N., \& Matías, K. (2016). Evolución del Sector Manufacturero ecuatoriano 2010-2013. Tipologías Estáticas y Dinámicas de la Manufactura. Quito, Pichincha, Ecuador: INEC Instituto Nacional de Estadísticas y Censos.

Gerdau. (6 de Mayo de 2016). Gerdau. Obtenido de Gerdau: https://www.gerdau.com/

Hernández Sampieri, R., \& Fernández Collado, C. (2003). Metodología de la Investigación. México: McGraw-Hill.

Historia de la Siderurgia minerales de hierro obtención del acero. (2014). Obtenido de Historia y Biografías: https://historiaybiografias.com/siderurgia/

Historia de la Siderurgia Minerales de Hierro Obtención del Acero. (21 de Diciembre de 2014). Historia y Biografias. Obtenido de Historia y Biografías: http://historiaybiografias.com/siderurgia/ Ibañez, J. (1992). La guerra incruenta entre cuantitativas y cualitativas.

Merino, T. (2011). Como escribir documentos científicos (Parte 3). Artículo de revisión. Salud en Tabasco, 17, 36-40. Obtenido de http://www.redalyc.org/articulo.oa?id=48721182006

Morales, N. (2016). Lifeder. Obtenido de https://www.lifeder.com/investigacion-exploratoria/ Motorgiga. (1998). Motorgiga. Recuperado el 8 de abril de 2017, de https://diccionario.motorgiga.com/diccionario/s-a-e-definicion-significado/gmx-niv15con195472.htm

Proecuador. (2013). Analìsis del Sector Metalmecànico. Instituto de Promoción de Exportación e Inversiones, Dirección de Inteligencia Comercial e Inversiones. Quito: Proecuador.

Reverso Diccionario. (2017). Obtenido de http://diccionario.reverso.net/espanoldefiniciones/siderurgia

Rodríguez, J. A. (2007). La importancia del reciclado del metal. Interempresas, Metalmecánica. 
Perfil ecuatoriano de las empresas metalmecánicas

Schwab, K. (2016). La cuarta revolución industrial. Madrid: DEBATE.

Shuttleworth, M. (2008). Diseño de investigación descriptiva. https://explorable.com/es/diseno-deinvestigacion-descriptiva.

Tendenzias. (2017). Sobrehistoria. Obtenido de https://sobrehistoria.com/todo-sobre-la-revolucionindustrial/ 